\title{
The QCD Phase Transition at finite Isospin
}

\author{
Rainer Stiele $^{a b}$ and Jürgen Schaffner-Bielich ${ }^{a b c}$ \\ ${ }^{a}$ Institut für Theoretische Physik, Universität Heidelberg, \\ Philosophenweg 16, 69120 Heidelberg, Germany \\ ${ }^{b}$ ExtreMe Matter Institute EMMI, GSI, Planckstraße 1, 64291 Darmstadt, Germany \\ ${ }^{c}$ Institut für Theoretische Physik, Goethe-Universität Frankfurt, \\ Max-von-Laue-Straße 1, D-60438, Frankfurt am Main, Germany \\ E-mail: R.Stieledthphys.uni-heidelberg.de, \\ Schaffnerdastrouni-frankfurt.de
}

Dense matter as produced in core collapse supernovae or in heavy ion collisions with $\mathrm{Pb}$ or $\mathrm{Au}$ beams is highly asymmetric in isospin. Also in the early universe the poorly constrained lepton asymmetry might be orders of magnitude larger than the baryon asymmetry allowing for a large isospin asymmetry. Hence, for the search of signatures of the QCD phase transition under these extreme conditions, the dependence on finite net isospin densities should be taken into account. We investigate the phase structure of strongly interacting matter within a Polyakov-quark-meson model that includes the physics of chiral symmetry breaking and restoration, as well as from the confinement-deconfinement phase transition. We present our results on the QCD phase diagram for different asymmetries in isospin.

Xth Quark Confinement and the Hadron Spectrum,

October 8-12, 2012

TUM Campus Garching, Munich, Germany 


\section{Polyakov-quark-meson model at finite isospin}

The goal for calculations with effective models is to find the minimum of the grand canonical potential $\Omega$ that is a function of the relevant order parameters and thermodynamic control parameters. For $2+1$ quark flavours and isospin asymmetric matter the order parameters of chiral symmetry are the chiral condensates $\sigma_{\mathrm{u}}, \sigma_{\mathrm{d}}$ and $\sigma_{\mathrm{s}}$ for the different quark sectors. In the gauge sector, the natural variables are the Polyakov loop $\Phi$ itself, and its conjugate $\bar{\Phi}$ that specify the free energy of quarks and antiquarks, respectively (e.g. [四-可]).

$$
\Omega\left(\sigma_{\mathrm{u}}, \sigma_{\mathrm{d}}, \sigma_{\mathrm{s}}, \Phi, \bar{\Phi} ; T, \mu_{f}\right)=U\left(\sigma_{\mathrm{u}}, \sigma_{\mathrm{d}}, \sigma_{\mathrm{s}}\right)+\mathcal{U}\left(\Phi, \bar{\Phi} ; T, \mu_{f}\right)+\Omega_{\mathrm{q} \overline{\mathrm{q}}}\left(\sigma_{\mathrm{u}}, \sigma_{\mathrm{d}}, \sigma_{\mathrm{s}}, \Phi, \bar{\Phi} ; T, \mu_{f}\right)
$$

The last term, $\Omega_{\mathrm{q} \overline{\mathrm{q}}}$ represents the contribution of the constituent quarks. It contains a minimal coupling to the gauge fields and a coupling to the mesons via a Yukawa-type term. The coupling with the mesons is translated into the masses of the quarks via spontaneous and explicit chiral symmetry breaking in the self-interaction potential of the mesons $U\left(\sigma_{\mathrm{u}}, \sigma_{\mathrm{d}}, \sigma_{\mathrm{s}}\right)$. Compared to previous studies e.g. in Refs. [[], [8] of isospin symmetric matter we have to generalise this contribution to distinguish chiral symmetry in the up and down quark sectors,

$$
\begin{aligned}
U\left(\sigma_{\mathrm{u}}, \sigma_{\mathrm{d}}, \sigma_{\mathrm{s}}\right)= & \frac{\lambda_{1}}{4}\left[\left(\frac{\sigma_{\mathrm{u}}^{2}+\sigma_{\mathrm{d}}^{2}}{2}\right)^{2}+\sigma_{\mathrm{s}}^{4}+\left(\sigma_{\mathrm{u}}^{2}+\sigma_{\mathrm{d}}^{2}\right) \sigma_{\mathrm{s}}^{2}\right]+\frac{\lambda_{2}}{4}\left(\frac{\sigma_{\mathrm{u}}^{4}+\sigma_{\mathrm{d}}^{4}}{4}+\sigma_{\mathrm{s}}^{4}\right) \\
& -\frac{c}{2 \sqrt{2}} \sigma_{\mathrm{u}} \sigma_{\mathrm{d}} \sigma_{\mathrm{s}}+\frac{m^{2}}{2}\left(\frac{\sigma_{\mathrm{u}}^{2}+\sigma_{\mathrm{d}}^{2}}{2}+\sigma_{\mathrm{s}}^{2}\right)-\frac{h_{\mathrm{ud}}}{2}\left(\sigma_{\mathrm{u}}+\sigma_{\mathrm{d}}\right)-h_{\mathrm{s}} \sigma_{\mathrm{s}}
\end{aligned}
$$

Since we want to conserve isospin symmetry of the vacuum, we imply the same explicit chiral symmetry breaking term for up and down quarks and therefore, the parameters are the same as in Refs. [प, [8].

The Polyakov loop potential $U(\Phi, \bar{\Phi} ; T)$ includes the physics of colour confinement as a potential energy for the expectation value of the Polyakov loop (e.g. [四, [1]). To include some aspects of the backreactions of quarks to the gauge sector, the effect of finite quark chemical potentials was parameterised in Ref. [[] as a running of the critical temperature in the gauge sector $T_{0}\left(\mu_{f}\right)$. We generalise this description to different chemical potentials for each quark sector.

$$
T_{0}\left(\mu_{f}\right)=m_{\tau} e^{-1 /\left(\alpha_{0} b\left(\mu_{f}\right)\right)} \quad \text { with } \quad b\left(\mu_{f}\right)=\bar{b}\left(N_{f}\right)-b_{\mu} \sum_{N_{f}} \frac{\mu_{f}^{2}}{m_{\tau}^{2}} \frac{\tilde{T}_{0}^{2}}{\tilde{T}_{0}^{2}+m_{f}^{2}},
$$

where

$$
\bar{b}\left(N_{f}\right)=\frac{1}{6 \pi}\left[11 N_{c}-2 \sum_{N_{f}} \frac{\tilde{T}_{0}^{2}}{\tilde{T}_{0}^{2}+m_{f}^{2}}\right] \quad \text { and } \quad b_{\mu} \simeq \frac{16}{\pi},
$$

and $\tilde{T}_{0}$ is the critical temperature for one massless flavour, $\tilde{T}_{0} \simeq 239 \mathrm{MeV}$.

\section{Results and discussion}

With the extensions described above we can extend the analysis of the phase structure and thermodynamics of QCD to finite isospin. Neglecting pion condensation so far, our results are 


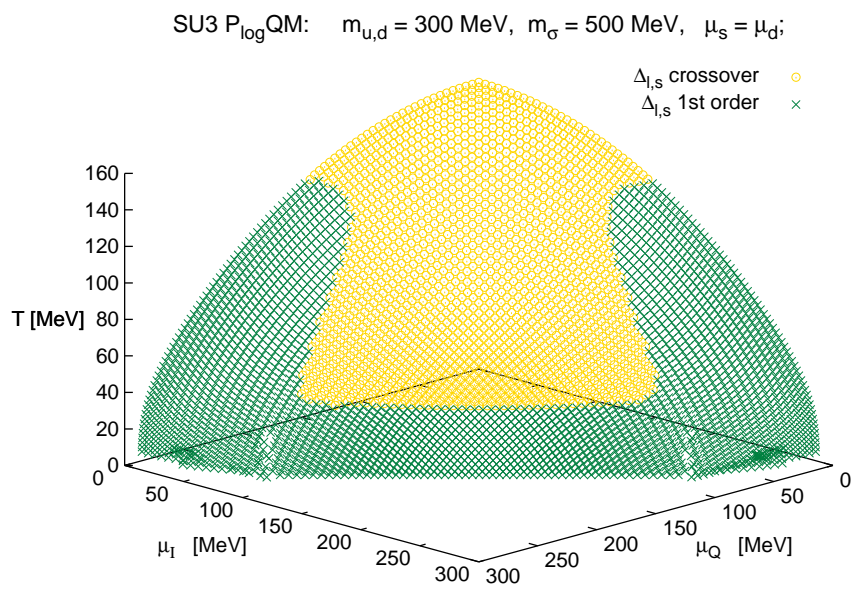

Figure 1: Three dimensional chiral phase diagram of the subtracted chiral condensate $\Delta_{\mathrm{l}, \mathrm{s}} . \quad \mu_{\mathrm{Q}}$ is defined as $\mu_{\mathrm{Q}}=\left(\mu_{\mathrm{u}}+\mu_{\mathrm{d}}\right) / 2$ and $\mu_{\mathrm{I}}$ as $\mu_{\mathrm{I}}=\left(\mu_{\mathrm{u}}-\mu_{\mathrm{d}}\right) / 2$. We consider $\beta$-equilibrium, so $\mu_{\mathrm{s}}=\mu_{\mathrm{d}}$ as it is the case in the early universe and supernovae. On the yellow surface the chiral transition is a crossover while on the green surface it is of first order.

strictly only valid up to $\mu_{\mathrm{I}} \leq m_{\pi} / 2$ [Q], besides the Silver Blaze problem [एర]]. In Fig. Wwe show the extension of the phase diagram to finite isospin. As in the two flavour studies with the PNJL model [ए]] and the Quark-Meson model with the functional renormalisation group [U]] the critical line at $T=0$ bends towards smaller $\mu_{\mathrm{Q}}$ and the critical endpoint towards larger $\mu_{\mathrm{Q}}$ with increasing isospin. Neglecting the coupling of the charged pions to the isospin, the phase diagram is symmetric in $\mu_{\mathrm{Q}}$ and $\mu_{\mathrm{I}}$ since the only dependence on the quark chemical potentials in Eq. ([L.Z) is quadratic. So, the pseudocritical temperature decreases as a function of the isospin at vanishing quark density as e.g. in Ref. [12].

In the remaining figures we show the evolution of the chiral condensates and the Polyakovloop variable. Figure (D) shows the common picture of a crossover transition at vanishing density and a discontinuous transition of first order at small temperature that is induced by the transition in the non-strange chiral sector.

Going from vanishing isospin shown in the left part of Fig. [3 to a finite isospin chemical potential as shown on the right of Fig. [ 3 the evolution of the chiral condensates in the up and down quark sectors splits. This effect becomes enhanced if we consider the evolution of the order parameters along $\mu_{\mathrm{Q}}=\mu_{\mathrm{I}}$, so at vanishing chemical potential of down quarks but increasing up quark chemical potential (Fig. (1). At finite isospin the chiral field $\sigma_{3}$ becomes non-zero that leads to the difference
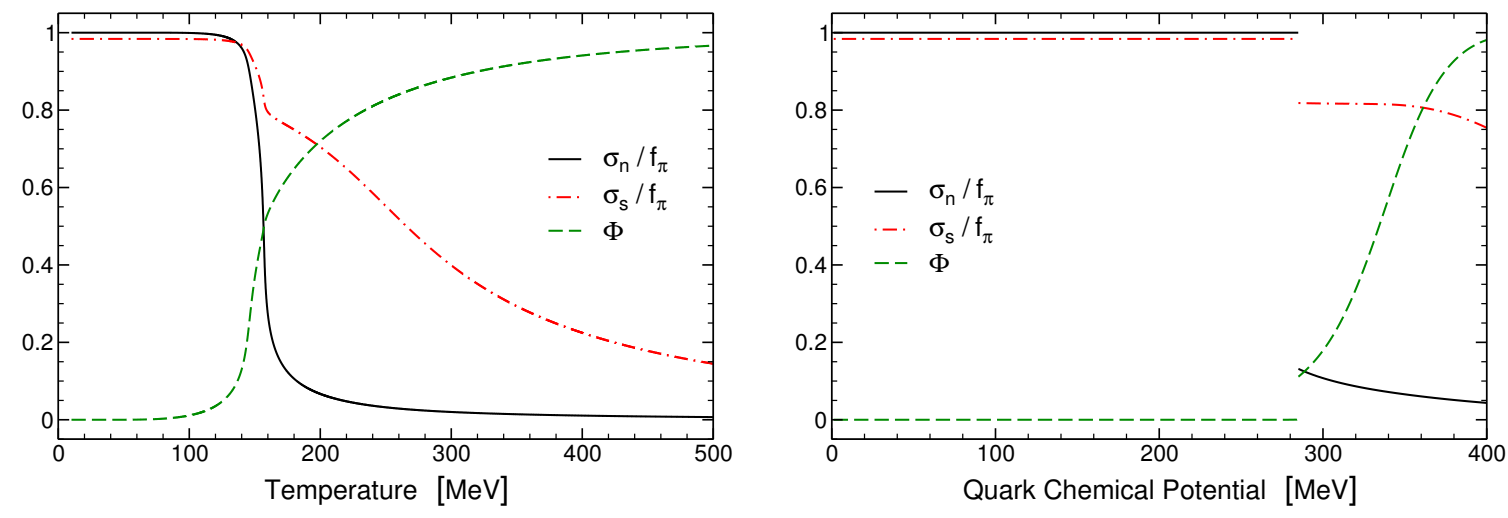

Figure 2: Evolution of the chiral condensates and the Polyakov loop with increasing temperature at vanishing density (left) and with increasing quark chemical potential at a temperature of $10 \mathrm{MeV}$ (right). 

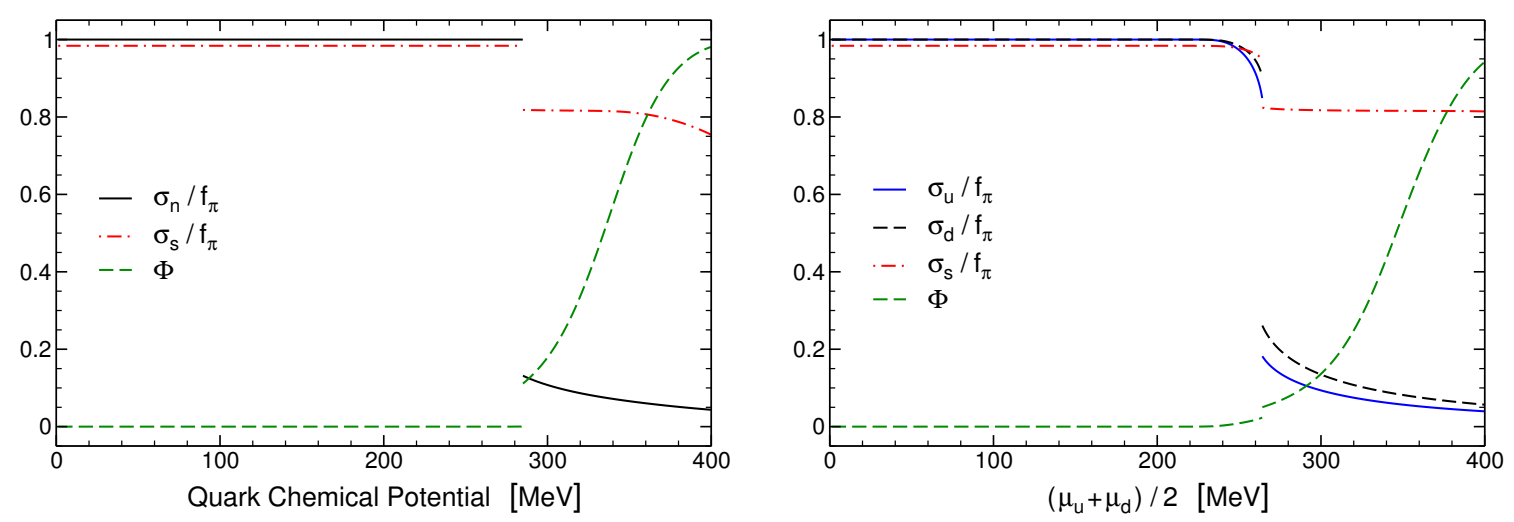

Figure 3: Evolution of the chiral condensates and the Polyakov loop at a temperature of $10 \mathrm{MeV}$ with increasing quark chemical potentials at vanishing isospin (left) and at a isospin chemical potential of $\mu_{\mathrm{I}}=$ $\left(\mu_{\mathrm{u}}-\mu_{\mathrm{d}}\right) / 2=65 \mathrm{MeV}$.

of the chiral condensates in the up-quark sector $\left(\sigma_{\mathrm{u}}=\sigma_{\mathrm{n}}+\sigma_{3}\right)$ and of the down quarks $\left(\sigma_{\mathrm{d}}=\right.$ $\left.\sigma_{\mathrm{n}}-\sigma_{3}\right)$ around the average non-strange chiral condensate $\sigma_{\mathrm{n}}=\left(\sigma_{\mathrm{u}}+\sigma_{\mathrm{d}}\right) / 2$ [प, [3]]. Since at finite isospin the quark chemical potential of the down quarks is reduced $\left(\mu_{\mathrm{d}}=\mu_{\mathrm{Q}}-\mu_{\mathrm{I}}\right)$ the phase

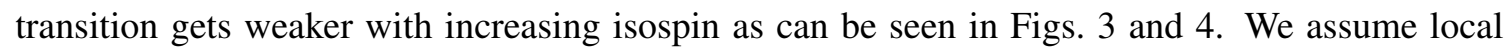
$\beta$-equilibrium with respect to weak flavour-mixing interactions so that $\mu_{\mathrm{s}}=\mu_{\mathrm{d}}$ as it is the case for the QCD transition in the early universe or in supernovae ${ }^{1}$. This implies that in Fig. $\mathbb{\text { ⿴囗十 }}$ not only the down quark chemical potential vanishes but also $\mu_{\mathrm{s}}=0$ so that the transition in the strange chiral sector is only induced due to the coupling to the chiral condensate of the up quarks and the chiral condensate in the strange sector tends to stay constant.

This work can be extended to include further particle asymmetries and to study its implications on the QCD phase transition [144].
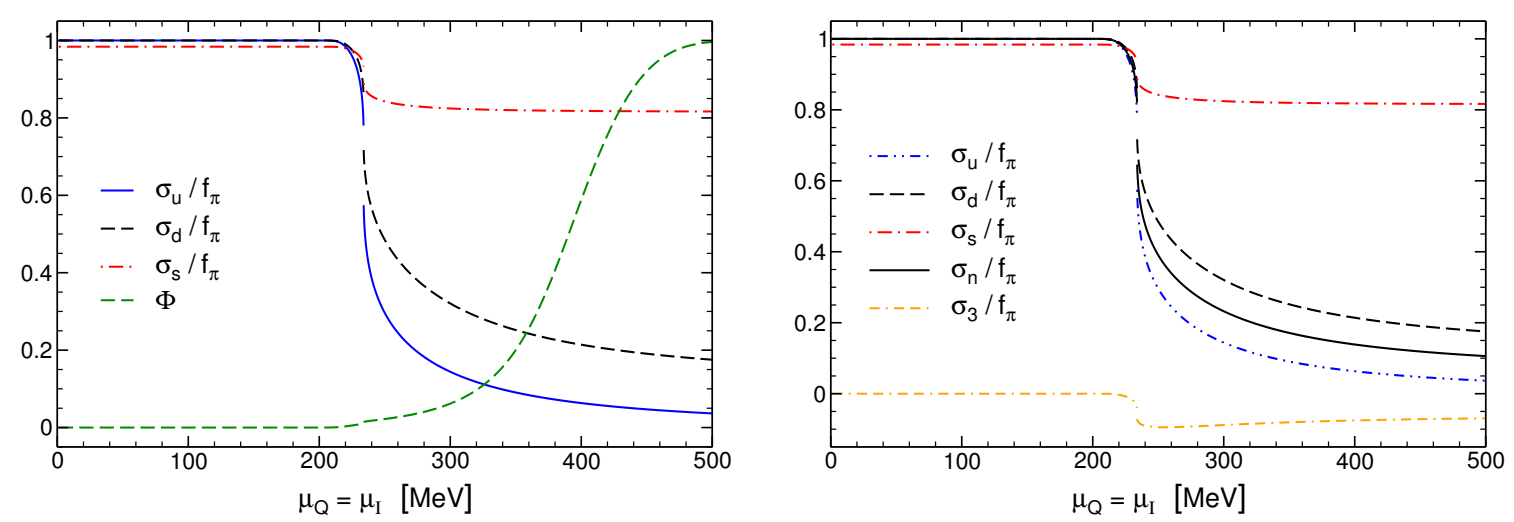

Figure 4: Evolution of the chiral condensates and the Polyakov loop at a temperature of $10 \mathrm{MeV}$ with increasing quark chemical potential of the up quark at vanishing quark chemical potentials of down and strange quarks, so along $\mu_{\mathrm{Q}}=\mu_{\mathrm{I}}$. The finite value of the condensate $\sigma_{3}$ (shown additionally on the right) induces the splitting of the chiral condensates of up and down quarks and their deviation from the average non-strange condensate.

\footnotetext{
${ }^{1}$ However, in heavy ion collisions no net strangeness can be produced what implies $\mu_{\mathrm{s}}=0$.
} 


\section{Acknowledgments}

This work is supported by BMBF under grants FKZ 06HD9127 and 05P12VHCTG, by the German Research Foundation (DFG) within the framework of the excellence initiative through the Heidelberg Graduate School of Fundamental Physics (HGSFP) and through the Helmholtz Graduate School for Heavy-Ion Research (HGS-HIRe) and the Graduate Program for Hadron and Ion Research (GP-HIR) by the Gesellschaft für Schwerionenforschung (GSI), Darmstadt and the Alliance Program of the Helmholtz Association HA216/EMMI.

\section{References}

[1] S. Roessner, C. Ratti, and W. Weise, "Polyakov loop, diquarks and the two-flavour phase diagram," Phys. Rev. D 75 (2007) 034007, arXiv:hep-ph/0609281 [hep-ph].

[2] B.-J. Schaefer, J. M. Pawlowski, and J. Wambach, “The Phase Structure of the Polyakov-Quark-Meson Model," Phys. Rev. D 76(2007) 074023, arXiv:0704.3234 thep-ph.

[3] B. W. Mintz, R. Stiele, R. O. Ramos, and J. Schaffner-Bielich, "Nucleation of quark matter in the PQM model," AIP Conf. Proc. 1520 (2012) 370-372.

[4] B. W. Mintz, R. Stiele, R. O. Ramos, and J. Schaffner-Bielich, "Phase Diagram and Surface Tension in the 3-flavor Polyakov-Quark-Meson Model," Phys. Rev. D 87 (2013) 036004, arXiv:1212.1184 [hep-ph].

[5] L. M. Haas, R. Stiele, J. Braun, J. M. Pawlowski, and J. Schaffner-Bielich, "Improved Polyakov-loop potential for effective models from functional calculations," Phys. Rev. D 87(2013) 076004, \begin{tabular}{l} 
arXiv:1302.1993 \\
\hline hep-ph
\end{tabular}

[6] R. Stiele, L. M. Haas, J. Braun, J. M. Pawlowski, and J. Schaffner-Bielich, "QCD thermodynamics of

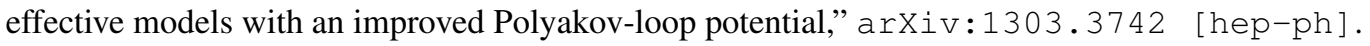

[7] J. T. Lenaghan, D. H. Rischke, and J. Schaffner-Bielich, "Chiral symmetry restoration at nonzero temperature in the SU(3)(r) x SU(3)(1) linear sigma model," Phys. Rev. D 62 (2000) 085008, arXiv:nucl-th/0004006 nucl-th.

[8] B.-J. Schaefer and M. Wagner, "The Three-flavor chiral phase structure in hot and dense QCD matter," Phys. Rev. D79(2009) 014018, arXiv:0808.1491

[9] D. Son and M. A. Stephanov, "QCD at finite isospin density," Phys. Rev. Lett. 86(2001) 592-595, arXiv:hep-ph/0005225 [hep-ph].

[10] K. Kamikado, N. Strodthoff, L. von Smekal, and J. Wambach, "Fluctuations in the quark-meson model for QCD with isospin chemical potential," Phys. Lett. B 718 (2013) 1044-1053, arXiv:1207.0400 hep-ph].

[11] T. Sasaki, Y. Sakai, H. Kouno, and M. Yahiro, "QCD phase diagram at finite baryon and isospin chemical potentials," Phys. Rev. D 82(2010) 116004, arXiv:1005.0910 [hep-ph].

[12] E. Fraga, L. Palhares, and C. Villavicencio, "Quark mass and isospin dependence of the deconfining critical temperature," Phys. Rev. D79(2009) 014021, arXiv:0810.1060 hep-ph].

[13] P. Kovacs and Z. Szep, "Influence of the isospin and hypercharge chemical potentials on the location of the CEP in the mu(B) - T phase diagram of the $\mathrm{SU}(3)(\mathrm{L}) \mathrm{x} \mathrm{SU}(3)(\mathrm{R})$ chiral quark model," Phys. Rev. D 77 (2008) 065016, arXiv:0710.1563 hep-ph]. 
[14] D. J. Schwarz and M. Stuke, "Lepton asymmetry and the cosmic QCD transition," VCAP 0911 (2009] D25, arXiv:0906.3434 [hep-ph]. 